\title{
Anatomía Morfométrica de Importancia Clínica del Atlas en Relación a la Estabilización del Complejo Atlantoaxial y Occipitocervical
}

\author{
Morphometric Anatomy of Clinical Importance of the Atlas in Relation \\ to the Stabilization of the Atlantoaxial and Occipitocervical Complex
}

Rodolfo Morales-Avalos ${ }^{1}$; Francisco Ismael Villarreal-Garcia ${ }^{1}$; Priscila Madelein Requena-Araujo ${ }^{2}$; Santos Guzmán-López ${ }^{2}$; Gabriela Sanchez-Mejorada ${ }^{3}$; Claudia Nallely Esparza-Hernandez²; Félix Vílchez-Cavazos ${ }^{1}$; Carlos Acosta-Olivo;

Víctor Manuel Peña-Martínez ${ }^{1}$ \& Rodrigo E. Elizondo-Omaña ${ }^{2}$

MORALES-AVALOS, R.; VILLARREAL-GARCIA, F. I.; REQUENA-ARAUJO, P. M.; GUZMÁN-LÓPEZ, S.; SANCHEZMEJORADA, G.; ESPARZA-HERNANDEZ, C. N.; VÍLCHEZ-CAVAZOS, F.; ACOSTA-OLIVO, C.; PEÑA-MARTÍNEZ, V. M. \& ELIZONDO-OMAÑA, R. E. Anatomía morfométrica de importancia clinica del atlas en relación a la estabilización del complejo atlantoaxial y occipitocervical. Int. J. Morphol., 37(1):98-103, 2019.

RESUMEN: Evaluar de manera integral los parámetros morfométricos de la vértebra atlas $(\mathrm{C} 1)$ en la población mexicana de relevancia en la realización de procedimientos quirúrgicos de la unión craneocervical con el fin de proveer datos cuantitativos indispensables para su realización. Para este estudio se utilizaron un total de 576 vértebras $\mathrm{C} 1$ secas de población mexicana contemporánea. Se realizaron 11 mediciones respecto a la morfología de $\mathrm{C} 1$. Las mediciones se efectuaron bilateralmente utilizando un vernier digital milimétrico con una precisión de 0,01 milímetros y se aplicó un análisis estadístico. Un total de 576 vértebras atlas (C1), fueron medidas de manera bilateral, todas nuestras mediciones se reportaron en milímetros. El promedio del ancho de las masas lateral fue de $14,87 \mathrm{~mm}$ $\pm 1,38 \mathrm{~mm}$. El promedio de la altura de las masas laterales fue de 4,05 $\mathrm{mm} \pm 0,93 \mathrm{~mm}$. El promedio del ancho del foramen transverso fue de $5,93 \mathrm{~mm} \pm 0,98 \mathrm{~mm}$. El promedio del largo del foramen transverso fue de $6,96 \mathrm{~mm} \pm 0,98 \mathrm{~mm}$. El promedio de la altura del foramen transverso fue de $5,76 \mathrm{~mm} \pm 1,31 \mathrm{~mm}$. El promedio del ancho del surco de la arteria vertebral fue de $18,87 \mathrm{~mm} \pm 1,3 \mathrm{~mm}$. El promedio de la distancia entre la línea media y el margen medial del surco de la arteria vertebral sobre la superficie anterior del arco posterior de C1 en su cara superior fue de 12,47 $\mathrm{mm} \pm 3,14 \mathrm{~mm}$. El promedio de la distancia entre la línea media y el margen medial del surco de la arteria vertebral sobre la superficie posterior del arco posterior de $\mathrm{C} 1$ en su cara superior fue de 18,75 $\mathrm{mm} \pm 3,94 \mathrm{~mm}$. El promedio de la distancia entre la línea media y el margen lateral del surco de la arteria vertebral sobre la superficie anterior del arco posterior de C1 en su cara superior fue de $19,7 \mathrm{~mm} \pm 2,52 \mathrm{~mm}$. El promedio de la distancia entre la línea media y el margen lateral del surco de la arteria vertebral sobre la superficie posterior del arco posterior de $\mathrm{C} 1 \mathrm{en}$ su cara superior fue de $29,52 \mathrm{~mm} \pm 3,23$. Nuestro estudio demuestra que las mediciones realizadas en nuestra población presentan diferencias significativas respecto a lo reportado actualmente en la literatura. Es necesario contar con un conocimiento de la morfología vertebral en nuestra población para disminuir la ventana de error al realizar procedimientos quirúrgicos que involucren este segmento.

PALABRAS ClAVE: Atlas; Morfometría; Anatomía; Pedículo; Masas laterales.

\section{INTRODUCCIÓN}

La primer vértebra cervical o atlas $(\mathrm{C} 1)$, cuenta con características únicas que la hacen diferente del resto de las vértebras cervicales. La más importante de ellas, es la ausencia de cuerpo vertebral. Consiste en dos masas laterales conectadas por 2 anillos, uno anterior y otro posterior. El anillo posterior es convexo y cuenta con un surco en su porción posterior el cual aloja la arteria vertebral y la rama de C1. La arteria vertebral cursa por el surco vertebral al salir del foramen transverso de $\mathrm{C} 1$ y toma una dirección medial terminando lateral al canal espinal (Francis, 1955).

\footnotetext{
${ }^{1}$ Servicio de Ortopedia y Traumatología, Hospital Universitario “Dr. José Eleuterio González”, Universidad Autónoma de Nuevo León (U.A.N.L.), Monterrey, Nuevo León, México.

${ }^{2}$ Departamento de Anatomía Humana, Facultad de Medicina, Universidad Autónoma de Nuevo León (U.A.N.L.), Monterrey, Nuevo León, México.

${ }^{3}$ Laboratorio de Antropología Física, Departamento de Anatomía, Facultad de Medicina, Universidad Nacional Autónoma de México (U.N.A.M.), Ciudad de México, México.
} 
Debido a la cercana relación entre las estructuras mencionadas y la importancia de estas en la cirugía de la unión craneocervical, es necesario el conocimiento de la morfología del atlas. La cirugía de unión craneocervical es un área importante de la cirugía de columna. Implica una variedad de procedimientos descompresivos y estabilizadores, que requieren conocimiento sobre la anatomía morfológica, neurovascular y musculoesquelética de esta región.

Se han descrito diversas técnicas de fusión de $\mathrm{C} 1$ y $\mathrm{C} 2$, que incluyen técnicas de alambrado posterior y fijación transarticular con tornillos a través de la pars interarticular del axis (C2) en la masa lateral de $\mathrm{C} 1$. Estas técnicas tienen limitaciones que pueden superarse mediante tornillos a las masas laterales de $\mathrm{C} 1$ cuya aplicación requiere un alto conocimiento de las características morfométricos del atlas y sus variantes (Christensen et al., 2007).

Durante los últimos años, los tornillos en C1 han ganado una gran popularidad, y varias series de casos han corroborado la seguridad de esta técnica (Goel, 2002). Las ventajas de esta técnica incluyen el hecho de que el gran tamaño de la masa lateral $\mathrm{C} 1$ hace que el procedimiento sea más seguro comparado con la colocación de tornillos transarticulares entre $\mathrm{C} 1$ y C2, lo que hace que la colocación de los tornillos sea factible en casi todos los pacientes (Dong et al., 2003).

Las variaciones genéticas y étnicas son características de la anatomía de la columna. Estas variaciones pueden requerir una modificación instrumental o hacer que la técnica sea inadecuada en algunos grupos étnicos debido a variaciones anatómicas incompatibles.

Al existir variaciones morfométricas entre poblaciones, es indispensable la elaboración de estudios morfométricos de dicha vertebra para evitar errores durante el procedimiento quirúrgico los cuales, debido al nivel, podrían tener un gran impacto en el paciente.

Se desconoce información cuantitativa sobre la morfología del atlas en la población mexicana. El objetivo del presente estudio fue determinar las características morfométricas de relevancia quirúrgica de la vértebra atlas en las técnicas más utilizadas en la actualidad para la estabilización quirúrgica de la unión occipitocervical y del complejo atlantoaxial.

\section{MATERIAL Y MÉTODO}

Estudio observacional, transversal y descriptivo. Se realizaron 11 mediciones respecto a la morfología de $\mathrm{C} 1$.
Las mediciones fueron realizadas en 576 vértebras C1 secas de población mexicana, obtenidas de los Departamentos de Anatomía Humana de la Facultad de Medicina y Facultad de Odontología de la UANL y el Laboratorio de Antropología Física del Departamento de Anatomía Humana de la Facultad de Medicina de la UNAM. Se excluyeron aquellas piezas en las que se observó daño o anormalidades evidentes en su estructura. Las mediciones de las vértebras se efectuaron bilateralmente utilizando un vernier digital milimétrico con una precisión de 0,01 milímetros (Mitutoyo Digimatic w/Absolute Encoders- Series 500). Todas las mediciones se reportaron en milímetros.

Los siguientes son los parámetros morfométricos evaluados en el presente estudio de mayor relevancia clínica para la estabilización occipitocervical y atlantoaxial mediante los distintos abordajes quirúrgicos existentes:

1. Altura AT. Altura del foramen transverso de C1: distancia entre el margen superior e inferior del foramen transverso de $\mathrm{C} 1$ (Fig. 1A).

2. Ancho AT. Anchura del foramen transverso del atlas: Distancia entre el margen medial y lateral del foramen transverso de C1 (Fig. 1B).

3. Ancho SAV. Anchura del surco de la arteria vertebral en C1: Distancia entre el margen lateral y medial del surco de la arteria vertebral en la cara superior del arco posterior de $\mathrm{C} 1$ (Fig. 1C).

4. Largo AT. Largo del foramen transverso de C1: Distancia entre el margen anterior y posterior del foramen transverso de C1 (Fig. 1D).

5. D/BLML-BMAT. Distancia entre el margen lateral de la zona de entrada del tornillo en las masas laterales y el margen medial del foramen transverso de $\mathrm{C} 1$ (Fig. 2A).

6. D/LMBL SAVa. Distancia entre la línea media y el margen lateral del surco de la arteria vertebral sobre la superficie anterior del arco posterior de $\mathrm{C} 1$ en su cara superior (Fig. 2B).

7. D/LMBL SAVp. Distancia entre la línea media y el margen lateral del surco de la arteria vertebral sobre la superficie posterior del arco posterior de $\mathrm{C} 1$ en su cara superior (Fig. 2C).

8. D/LMBM SAVp. Distancia entre la línea media y el margen medial del surco de la arteria vertebral sobre la superficie posterior del arco posterior de $\mathrm{C} 1$ en su cara superior (Fig. 2D). 

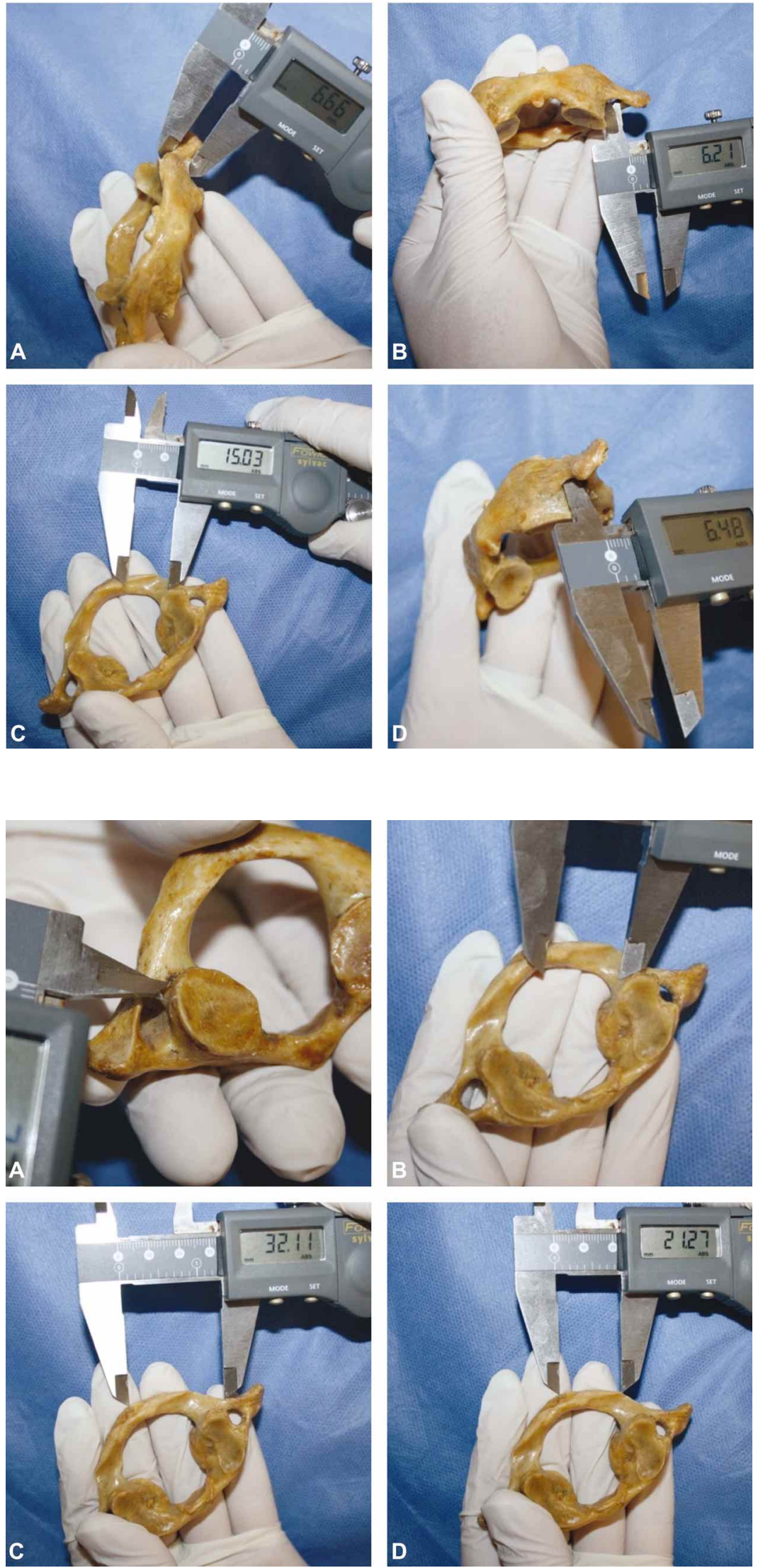

Fig. 1. (A) Altura AT (Altura del foramen transverso de C1) Distancia entre el borde superior e inferior del foramen transverso de C1. (B) Ancho AT (Ancho del foramen transverso) Distancia entre el borde medial y lateral del foramen transverso de C1. (C) Ancho SAV (Ancho del surco de la arteria vertebral de C1) Distancia entre el borde lateral y medial del surco de la arteria vertebral en la cara superior del arco posterior de C1. (D) Largo AT (Largo del foramen transverso de C1) Distancia entre el borde anterior y posterior del foramen transverso de $\mathrm{C} 1$.

Fig. 2. (A) D/BLML-BMAT (Distancia entre el borde lateral de la zona de entrada del tornillo en las masas laterales y el borde medial del foramen transverso de C1). (B) D/LMBL SAVa (Distancia entre la línea media y el borde lateral del surco de la arteria vertebral sobre la superficie anterior del arco posterior de $\mathrm{C} 1$ en su cara superior). (C) D/LMBL SAVp (Distancia entre la línea media y el borde lateral del surco de la arteria vertebral sobre la superficie posterior del arco posterior de $\mathrm{C} 1$ en su cara superior). (D) D/LMBM SAVp (Distancia entre la línea media y el borde medial del surco de la arteria vertebral sobre la superficie posterior del arco posterior de $\mathrm{C} 1$ en su cara superior). 

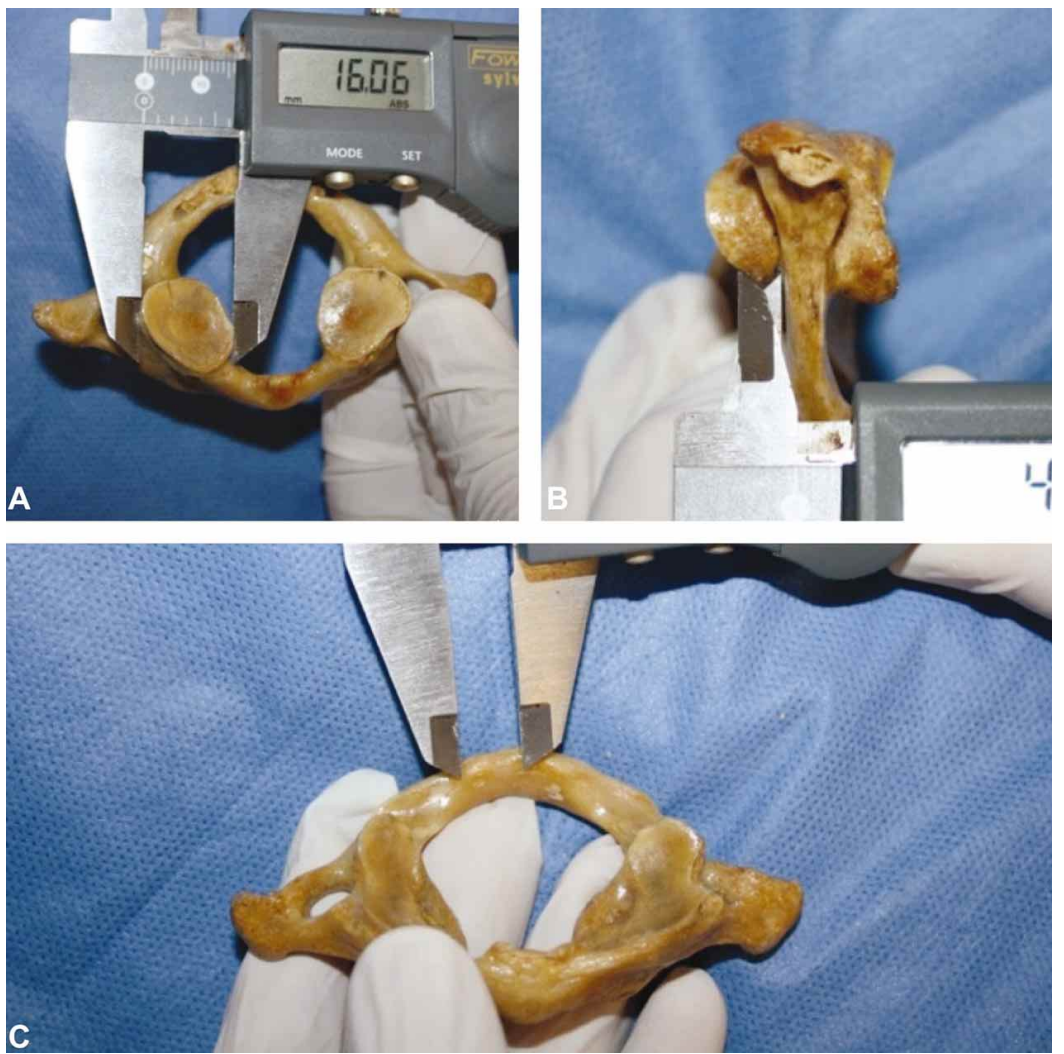

Fig. 3. (A) Ancho ML (Ancho de la zona de entrada del tornillo en las masas laterales) Distancia entre el borde medial y lateral de la zona de entrada del tornillo en las masas laterales. (B) Altura ML (Altura de la zona de entrada del tornillo en las masas laterales) Distancia entre el borde superior e inferior de la zona de entrada del tornillo en las masas laterales. (C) D/LMBM SAVa (Distancia entre la línea media y el borde medial del surco de la arteria vertebral sobre la superficie anterior del arco posterior de $\mathrm{C} 1$ en su cara superior).

9. Ancho ML. Ancho de la zona de entrada del tornillo en las masas laterales. Distancia entre el margen medial y lateral de la zona de entrada del tornillo en las masas laterales el cual es definido como la porción de las masas laterales por debajo del arco posterior de C1 (Fig. 3A).

10. Altura ML. Altura de la zona de entrada del tornillo en las masas laterales. Distancia entre el margen superior e inferior de la zona de entrada del tornillo en las masas laterales el cual es definido como la porción de las masas laterales por debajo del arco posterior de C1 (Fig. 3B).

11.D/LMBM SAVa. Distancia entre la línea media y el margen medial del surco de la arteria vertebral sobre la superficie anterior del arco posterior de $\mathrm{C} 1$ en su cara superior (Fig. 3C).

Para cada una de las mediciones se tomaron de referencia puntos anatómicos específicos de cada vértebra, ya realizadas las mediciones, se determinó la media y desviación estándar para cada parámetro.
Análisis Estadístico. Fue realizado utilizando el programa computacional Microsoft Excel 2015 para Windows XP. Se determinó la media, desviación estándar, resultado mínimo y resultado máximo para cada parámetro morfométrico estudiado, todos los resultados son expresados en milímetros.

Previo a la realización de las morfometrías se llevo a cabo un análisis interobservador para la estandarización de la técnica y los puntos de medición utilizando una prueba de $t$ student de dos colas para determinar la significancia de las diferencias entre las mediciones de los dos observadores, considerando como significativo un valor de $\mathrm{p}$ inferior a 0,05 .

Consideraciones Éticas. El presente protocolo fue aprobado por el Comité de Ética y el Comité de Investigación de la Facultad de Medicina de la Universidad Autónoma de Nuevo León. No existen ganancias financieras o comerciales por la realización del presente estudio, por lo que los autores declaran no tener ningún tipo de conflicto de interés.

\section{RESULTADOS}

Se analizaron un total de 576 vértebras C1 de edad y géneros desconocidos (1.152 mediciones de cada parámetro y un total de 12.672 mediciones entre todos los parámetros morfométricos de manera bilateral). Para cada vértebra se determinaron los promedios y desviaciones estándar de cada una de las variables a medir.

El resultado del análisis interobservador con una submuestra de 50 vertebras realizado para la estandarización de la técnica de medición arrojo valores no significativos en todos los casos por lo que consideramos que la técnica se estandarizó de manera correcta (valores de p entre 0,44 y 0,82 en las distintas 11 morfometrías. Se pueden observar las mediciones realizadas en la Tabla I. 
MORALES-AVALOS, R.; VILLARREAL-GARCIA, F. I.; REQUENA-ARAUJO, P. M.; GUZMÁN-LÓPEZ, S.; SANCHEZ-MEJORADA, G.; ESPARZA-HERNANDEZ, C. N.; VílCHEZ-CAVAZOS, F.; ACOSTA-OLIVO, C.; PEÑA-MARTíNEZ, V. M. \& ELIZONDO-OMAÑA, R. E. Anatomía morfométrica de importancia clinica del atlas en relación a la estabilización del complejo atlantoaxial y occipitocervical. Int. J. Morphol., 37(1):98-103, 2019.

Tabla I. Medidas realizadas en 576 vértebras C1. Mediciones reportadas en milímetros.

\begin{tabular}{lcccccccccc}
\hline & ANCHO & ALTURA & ANCHO & LARGO & ALTURA & ANCHO & D/LMBM & D/LMBM & D/LMBL & D/LMBL \\
& ML & ML & AT & AT & AT & SAV & SAVa & SAVp & SAVa & SAVb \\
\hline UEDIA & 14.87 & 4.05 & 5.93 & 6.96 & 5.76 & 18.87 & 12.47 & 18.75 & 19.7 & 29.52 \\
).E. & 1.38 & 0.93 & 0.98 & 0.98 & 1.31 & 1.3 & 3.14 & 3.94 & 2.52 & 3.23 \\
\hline
\end{tabular}

Ancho ML (Anchura de masas laterales), Altura ML (Altura de masas laterales), Ancho AT (Ancho del foramen transverso), Largo AT (Largo del foramen transverso), Altura AT (Altura del foramen transverso), Ancho SAV (Ancho del surco de la arteria), D/LMBM SAVa (Distancia entre la línea media y el borde medial del surco de la arteria vertebral sobre la superficie anterior del arcoposterior de C1 en su cara superior), D/LMBM SAVp (Distancia entre la línea media y el borde medial del surco de la arteria vertebral sobre la superficie posterior del arco posterior de C1 en su cara superior), D/LMBL SAVa (Distancia entre la línea media y el borde lateral del surco de la arteria vertebral sobre la superficie anterior del arco posterior de C1 en su cara superior, D/LMBL SAVp (Distancia entre la línea media y el borde lateral del surco de la arteria vertebral sobre la superficie posterior del arco posterior de C1 en su cara superior.

Podemos observar que el promedio del ancho de las masas lateral fue de $14,87 \mathrm{~mm} \pm 1,38$. El promedio de la altura de las masas laterales fue de $4,05 \mathrm{~mm} \pm 0,93$. El promedio del ancho del foramen transverso fue de $5,93 \mathrm{~mm} \pm$ 0,98 . El promedio del largo del foramen transverso fue de $6,96 \mathrm{~mm} \pm 0,98$. El promedio de la altura del foramen transverso fue de $5,76 \mathrm{~mm} \pm 1,31$. El promedio del ancho del surco de la arteria vertebral fue de $18,87 \mathrm{~mm} \pm 1,30$. El promedio de la distancia entre la línea media y el margen medial del surco de la arteria vertebral sobre la superficie anterior del arco posterior de $\mathrm{C} 1$ en su cara superior fue de $12,47 \mathrm{~mm} \pm 3,14$. El promedio de la distancia entre la línea media y el margen medial del surco de la arteria vertebral sobre la superficie posterior del arco posterior de $\mathrm{C} 1$ en su cara superior fue de $18,75 \mathrm{~mm} \pm 3,94$. El promedio de la distancia entre la línea media y el margen lateral del surco de la arteria vertebral sobre lasuperficie anterior del arco posterior de $\mathrm{C} 1 \mathrm{en}$ su cara superior fue de $19,7 \mathrm{~mm} \pm 2,52$. El promedio de la distancia entre la línea media y el margen lateral del surco de la arteria vertebral sobre la superficie posterior del arco posterior de $\mathrm{C} 1$ en su cara superior fue de $29,52 \mathrm{~mm} \pm 3,23$.

\section{DISCUSIÓN}

Las características anatómicas macroscópicas de la vértebra atlas han sido bien descritas por generaciones de anatomistas (Monu et al., 1987). En 1952, Francis reportó el diámetro total anterior y posterior de 285 vértebras atlas. Doherty \& Heggeness (1994) reportaron el rango y la variabilidad de las dimensiones externas del atlas y el grosor cortical y la distribución trabecular de esta vértebra.

La superioridad de la estabilidad biomecánica de la fijación con tornillo transpedicular en la región cervical, así como en la región lumbar y torácica actualmente es una técnica ampliamente aceptada (Abumi \& Kaneda, 1997).
Diferentes procedimientos de estabilización se llevan acabo en la unión occipitocervical, incluida la fijación con tornillos transarticulares en C1 y C2. Además de la necesidad de información sobre la ubicación de la arteria vertebral, esta cirugía requiere el conocimiento de la distancia entre el margen anterior de la masa lateral de $\mathrm{C} 1$ y el tubérculo anterior de $\mathrm{C} 1$. Esta distancia debe tenerse en cuenta durante la fijación con tornillo transarticular entre C1 y C2. Un tornillo demasiado largo puede penetrar en los tejidos blandos anteriores y ocasionar lesión de los mismos (Grob et al., 1999).

La falta de cuerpo vertebral y una forma anular diferencian el atlas de las otras vértebras cervicales. El anillo de $\mathrm{C} 1$ consiste en un arco anterior y otro posterior, dos masas laterales con sus facetas superior e inferior respectivamente. La ubicación de la arteria vertebral puede complicar los procedimientos descompresivos en esta región. El riesgo de lesión de la arteria vertebral durante la cirugía en esta región ha sido abordado por algunos autores (An et al., 1994).

Las técnicas de instrumentación posterior con tornillo en $\mathrm{C} 1$ incluyen la fijación con tornillo en la masa lateral de $\mathrm{C} 1$ y la fijación con tornillo pedicular en C1. Los tornillos de masa lateral $\mathrm{C} 1$ se insertan directamente a través de la base inferior del arco posterior. Los llamados tornillos pediculares $\mathrm{C} 1$ se colocan realmente a través del arco posterior o el "pedículo" de C1. Las trayectorias de los tornillos pediculares son más largas que las de los tornillos en la masa lateral $\mathrm{C} 1$; por lo tanto, la fijación con tornillo pedicular $\mathrm{C} 1$ puede tener una mejor fijación en el hueso que los tornillos en la masa lateral C1 (Resnick \& Benzel, 2002).

Nadery et al. (2003) y cols determinaron que el promedio de la distancia entre la línea media y el margen interno del surco de la arteria vertebral en su vista posterior fue de $15,03 \mathrm{~mm}$ y una $\mathrm{DE} \pm 1,22 \mathrm{~mm}$ y en nuestro estudio fue de $18,75 \mathrm{~mm}$ y una $\mathrm{DE} \pm 3,94 \mathrm{~mm}$. Al ser esta la única medición que presenta similitud a la medición en nuestro estudio, nos 
hace pensar que si existen diferencias morfológicas relevantes entre poblaciones (Naderi et al.).

Dong et al. realizaron un estudio de morfología donde determinaron que el ancho de la masa lateral del atlas es de $11,6 \mathrm{~mm}$ con una $\mathrm{DE} \pm 1,4 \mathrm{~mm}$. Estos resultados contrastan con nuestras mediciones ya que el promedio del ancho de la masa lateral en nuestra población fue de 14,87 $\mathrm{mm}$ con una $\mathrm{DE} \pm 1,38 \mathrm{~mm}$ (Dong et al.).

\section{CONCLUSIONES}

Nuestro estudio demuestra que las mediciones realizadas en nuestra población, si presentan diferencias significativas respecto a lo reportado actualmente en la literatura. Es necesario contar con un conocimiento de la morfología vertebral en nuestra población para disminuir la ventana de error al realizar procedimientos quirúrgicos que involucren este segmento.

MORALES-AVALOS, R.; VILLARREAL-GARCIA, F. I.; REQUENA-ARAUJO, P. M.; GUZMÁN-LÓPEZ, S.; SANCHEZ-MEJORADA, G.; ESPARZA-HERNANDEZ, C. N.; VÍlCHEZ-CAVAZOS, F.; ACOSTA-OLIVO, C.; PEÑAMARTÍNEZ, V. M. \& ELIZONDO-OMAÑA, R. E. Morphometric anatomy of clinical importance of the atlas in relation to the stabilization of the atlantoaxial and occipitocervical complex. Int. J. Morphol., 37(1):98-103, 2019.

SUMMARY: To evaluate in an integral way the morphometric parameters of the atlas $(\mathrm{C} 1)$ vertebra in the Mexican population of relevance in performing surgical procedures of the craniocervical junction in order to provide quantitative and essential data for its realization. For this study, a total of 576 dry $\mathrm{C} 1$ vertebrae of contemporary Mexican population were used. Eleven measurements were carried out regarding the morphology of $\mathrm{C} 1$. The measurements were made bilaterally using a millimeter digital vernier with an accuracy of 0.01 millimeters and a statistical analysis was applied. A total of 576 atlas vertebrae (C1) were measured bilaterally, all our measurements were reported in millimeters. The average width of the lateral masses was $14.87 \mathrm{~mm} \pm 1.38 \mathrm{~mm}$. The average height of the lateral masses was $4.05 \mathrm{~mm} \pm 0.93 \mathrm{~mm}$. The average width of the transverse foramen was $5.93 \mathrm{~mm} \pm 0.98 \mathrm{~mm}$. The average length of the transverse foramen was $6.96 \mathrm{~mm} \pm 0.98$ $\mathrm{mm}$. The average height of the transverse foramen was $5.76 \mathrm{~mm} \pm$ $1.31 \mathrm{~mm}$. The average width of the groove of the vertebral artery was $18.87 \mathrm{~mm} \pm 1.3 \mathrm{~mm}$. The average distance between the midline and the medial edge of the vertebral artery groove on the anterior surface of the posterior arch of $\mathrm{C} 1$ on its superior surface was 12.47 $\mathrm{mm} \pm 3.14 \mathrm{~mm}$. The average distance between the midline and the medial border of the vertebral artery groove on the posterior surface of the posterior arch of $\mathrm{C} 1$ on its upper face was $18.75 \mathrm{~mm} \pm 3.94$ $\mathrm{mm}$. The average distance between the midline and the lateral border of the vertebral artery groove on the anterior surface of the posterior arch of $\mathrm{C} 1$ on its superior surface was $19.7 \mathrm{~mm} \pm 2.52$ $\mathrm{mm}$. The average distance between the midline and the lateral border of the vertebral artery groove on the posterior surface of the posterior arch of $\mathrm{C} 1$ on its superior surface was $29.52 \mathrm{~mm} \pm 3.23$. Our study shows that the measurements made in our population present differences with respect to what is currently reported in the literature. It is necessary to have knowledge of vertebral morphology in our population to reduce the error window when performing surgical procedures involving this segment.

KEY WORDS: Atlas; Morphometry; Anatomy; Pedicle; Lateral masses.

\section{REFERENCIAS BIBLIOGRÁFICAS}

Abumi, K., \& Kaneda, K. Pedicle screw fixation for nontraumatic lesions of the cervical spine. Spine (Phila Pa 1976), 22(16):1853-63, 1997.

An, H. S.; Vaccaro, A.; Cotler, J. M. \& Lin, S. Spinal disorders at the cervicothoracic junction. Spine (Phila Pa 1976), 19(22):2557-64, 1994.

Christensen, D. M.; Eastlack, R. K.; Lynch, J. J.; Yaszemski, M. J. \& Currier, B. L. C1 anatomy and dimensions relative to lateral mass screw placement. Spine (Phila Pa 1976), 32(8):844-8, 2007.

Doherty, B. J. \& Heggeness, M. H. The quantitative anatomy of the atlas. Spine (Phila Pa 1976), 19(22):2497-500, 1994.

Dong, Y.; Hong, M. X.; Jianyi, L. \& Lin, M. Y. Quantitative anatomy of the lateral mass of the atlas. Spine (Phila Pa 1976), 28(9):860-3, 2003.

Francis, C. C. Dimensions of the cervical vertebrae. Anat. Rec., 122(4):6039, 1955.

Goel, A. C1-C2 pedicle screw fixation with rigid cantilever beam construct: case report and technical note. Neurosurgery, 51(3):853-4, 2002.

Grob, D.; Schütz, U. \& Plötz, G. Occipitocervical fusion in patients with rheumatoid arthritis. Clin. Orthop. Relat. Res., (366):46-53, 1999.

Monu, J.; Bohrer, S. P. \& Howard, G. Some upper cervical spine norms. Spine (Phila Pa 1976), 12(6):515-9, 1987.

Naderi, S.; Cakmakçi, H.; Acar, F.; Arman, C.; Mertol, T. \& Arda, M. N. Anatomical and computed tomographic analysis of $\mathrm{C} 1$ vertebra. Clin. Neurol. Neurosurg., 105(4):245-8, 2003.

Resnick, D. K. \& Benzel, E. C. C1-C2 pedicle screw fixation with rigid cantilever beam construct: case report and technical note. Neurosurgery, 50(2):426-8, 2002.

Dirección para correspondencia:

Dr. Rodolfo Morales Avalos

Servicio de Ortopedia y Traumatología

Hospital Universitario "Dr. José Eleuterio González"

Universidad Autónoma de Nuevo León (U.A.N.L.)

Ave. Madero s/n Col. Mitras Centro

Monterrey, Nuevo León

MÉXICO

Email: rodolfot59@hotmail.com

Recibido : 30-06-2018

Aceptado: $30-08-2018$ 